\title{
Effect of Plyometric Exercises on Developing Legs Explosive Powers on Front Acrobatic Routine for Floor Exercises (Front Handspring Followed By Front Full Twist Then Full Twist with Turn around Long Axis).
}

\section{Ashraf Abdel Aal}

Asst. Professor, Exercises and gymnastic training department, Faculty of physical Education for Men, Alexandria university. Egypt.

\begin{abstract}
During republic champion, researcher noticed the inability of many of players involved in Republic championships in performance of front routines, in particular, represented by the lack of linkage between the parts of the routine and thus lead to decrease in their grades, making them out of competition on floor exercise despite the high level of performance on other devices. So it was important to develop plyometric training program form of exercises similar with kinetic path for the acrobatic routine under study and identify its impact on its performance level.
\end{abstract}

\section{Research Objective:}

This research aims to effect of plyometric exercises on developing legs explosive powers on front acrobatic routine performance level and some physical elements relating to it.

\section{Conclusions :}

1-Using plyometric training has effective effect in developing physical abilities and explosive power specially of arms and legs.

2-Development of legs explosive power led to improvement in skill performance level.

3-Development of legs explosive power led to the development of other elements (power), motor range and flexibility for muscle involved in performance skills.

4-The presence of a high correlation between skill performance level and explosive power.

\section{Recommendations:}

1-There is necessity to pay attention to plyometric training in gymnastics selection of exercises cope with working muscle contractions methods in different skills performance.

2-Gymnastics trainers should pay interest to change training programs types and use plyometric training method; especially with juniors as it have positive impact on Gymnastics skill performance.

3-Conducting similar studies for different age stages and level for male and female players.

4-Plyometric training program to be included as part of physical education curricula.

5-Preparation of scalable training program suitable for application of different ages to contribute in improving skill performance level of gymnastics ground movements . 
Introduction:

esign of training programs in
gymnastics, specially skills needs specific expertise, as innovating new methods associated with the nature of skills performance. Therefore the researcher used the plyometric training to develop explosive power of the legs as one of specific methods associated with the nature of front acrobatic routine performance under discussion, which this type is based on muscle exercises while stretching (decentralized contraction) resulting in strong explosive contraction (central contraction) improves the level of development operations. (Medhat El Shafei, 2005: p526)

The front acrobatic routine is one of somersaults and turns group which is one of the main compound groups in floor exercises, and is considered a prerequisite in kinetic routine according to international law. (International Gymnastics Federation,2006: p36)

Egyptian Gymnastics Federation has developed some acrobatic front routines for various astages, including the routine under study, front handspring followed by front full twist then full twist with turn $360^{\circ}$ around long axis.

Through researcher's work as coach and judge, he noted the inability of many of players involved in Republic championships in performance of front routines, in particular, represented by the lack of linkage between the parts of the routine and thus lead to decrease in their grades, making them out of competition on floor exercise despite the high level of performance on other devices.

Researcher, therefore, developed plyometric training program in form of exercises similar with kinetic path for the acrobatic routine under study and identify its impact on its performance level.

\section{Research Objective:}

This research aims to effect of plyometric exercises on developing legs explosive powers on front acrobatic routine performance level and some physical elements relating to it.

\section{Research hypotheses:}

1- Training program had a positive effect on some physical elements related to routine under study.

2- Plyometric training program for developing legs explosive power positively affect front acrobatic routine performance level.

\section{Research procedures:}

This research was conducted during 3 months from June to August 2009, in Sport Establishment Club sports hall due to availability facilities required.

\section{Research Sample:}

The research sample was selected intentionally of from gymnasts at Alexandria area under 13 years age; 10 gymnasts participated in the study divided into two equal groups; one experimental and the other control, both were homogeneous in basic variables and some special physical elements and judges' assessment degrees of the level of skill performance.

\section{Pilot studies:}

\section{The first pilot study:}

The study was conducted during under 3 years juniors training to ensure the validity of devices used and see how much program exercises suite study sample. 
Table (1)

Statistical significance for basic variables and physical measurements and judges skill level degrees for overall study sample before experiment $\mathbf{n}=\mathbf{1 0}$

\begin{tabular}{|c|c|c|c|c|c|c|c|c|}
\hline \multicolumn{3}{|c|}{$\begin{array}{r}\text { Statistical indicators } \\
\text { skill measurements and tests }\end{array}$} & unit & Mean & SD & Skewness & Kurtosis & variation \\
\hline \multirow{3}{*}{\multicolumn{2}{|c|}{ Basic Variables }} & Age & Year & 12.83 & 0.51 & -0.67 & -1.18 & 3.97 \\
\hline & & Length & $\mathrm{Cm}$ & 142.9 & 2.08 & -0.3 & -1.62 & 1.45 \\
\hline & & Weight & Kg & 38.5 & 3.37 & -0.17 & -1.01 & 8.75 \\
\hline \multirow{8}{*}{ Power } & \multirow{3}{*}{ Arms } & $\begin{array}{c}\text { Arms bent from of } \\
\text { pivot / 10sec }\end{array}$ & repetition & 9.3 & 0.48 & 1.04 & -1.22 & 5.16 \\
\hline & & $\begin{array}{l}\text { Arms bent from } \\
\text { hanging / } 10 \mathrm{sec}\end{array}$ & repetition & 9.3 & 0.67 & -0.43 & -0.28 & 7.2 \\
\hline & & $\begin{array}{l}\text { Push ground with } \\
\text { arms from oblique } \\
\text { laying }\end{array}$ & repetition & 12.4 & 0.52 & 0.48 & -2.28 & 4.19 \\
\hline & \multirow{3}{*}{ Trunk } & Lift legs from lying & repetition & 10.5 & 0.53 & $\mathbf{0}$ & -2.57 & 5.04 \\
\hline & & $\begin{array}{c}\text { Lift legs from } \\
\text { hanging }\end{array}$ & repetition & 7.1 & 0.32 & 3.16 & 10 & 4.5 \\
\hline & & $\begin{array}{l}\text { Lift back and arms } \\
\text { from lying }\end{array}$ & repetition & 15.3 & 0.48 & 1.04 & -1.22 & 3.13 \\
\hline & \multirow{2}{*}{ Legs } & Long jump & $\mathbf{C m}$ & 209 & 0.82 & $\mathbf{0}$ & -1.39 & 0.39 \\
\hline & & Vertical jump & $\mathbf{C m}$ & 40.9 & 0.99 & 0.24 & -2.3 & 2.42 \\
\hline \multirow{4}{*}{$\begin{array}{l}\text { Motor } \\
\text { Range }\end{array}$} & & Dome & $\mathbf{C m}$ & 5.4 & 0.7 & 1.66 & 2.05 & 12.96 \\
\hline & \multicolumn{2}{|c|}{ Bent trunk down forward } & $\mathbf{C m}$ & 12.5 & 0.53 & $\mathbf{0}$ & -2.57 & 4.24 \\
\hline & \multicolumn{2}{|c|}{ Bent arms by degree } & Mark & 31.3 & 1.06 & -0.04 & -1.24 & 3.38 \\
\hline & \multicolumn{2}{|c|}{ Legs backward by degree } & Mark & 22.3 & 2.5 & 0.16 & -2.36 & 11.21 \\
\hline \multirow{3}{*}{$\begin{array}{l}\text { Skill } \\
\text { Level } \\
\text { Mark }\end{array}$} & \multicolumn{2}{|c|}{ Front straight full twist } & Mark & 3.9 & 0.74 & 0.17 & -0.73 & 18.97 \\
\hline & \multicolumn{2}{|c|}{$\begin{array}{l}\text { Straight full twist with turn } \\
\text { around long axis }\end{array}$} & Mark & 3.4 & 0.7 & 1.66 & 2.05 & 20.58 \\
\hline & \multicolumn{2}{|c|}{ Two skills linking } & Mark & 2.8 & 0.6 & 0.41 & -1.07 & 21.42 \\
\hline
\end{tabular}

Table (1) for statistical significance for basic variables and physical measurements and judges skill level degrees for overall study sample before experiment results reveal that skewness were between $( \pm 3)$ for whole sample which indicates that it is under normal cureve and that variances are in the accepted level for sample homogeneous. 
Table (2)

Statistical significance for basic variables and physical measurements and judges skill level degrees for experimental and control groups before experiment $\mathrm{n}=\mathbf{1 0}$

\begin{tabular}{|c|c|c|c|c|c|c|c|c|c|c|}
\hline \multicolumn{3}{|c|}{ Statistical indicators } & \multirow{2}{*}{ unit } & \multicolumn{2}{|c|}{$\begin{array}{l}\text { Experimental } \\
\text { group }\end{array}$} & \multicolumn{2}{|c|}{$\begin{array}{l}\text { Control } \\
\text { group }\end{array}$} & \multirow{2}{*}{ 芯 } & \multirow{2}{*}{$\mathbf{T}$} & \multirow{2}{*}{ 芯 } \\
\hline & & $\begin{array}{r}\text { Physical and } \\
\text { measurements and tests }\end{array}$ & & Mean & SD & Mean & SD & & & \\
\hline \multirow{3}{*}{\multicolumn{2}{|c|}{$\frac{\frac{y}{0}}{\frac{0}{0}}$}} & Age & Year & 12.69 & 0.53 & 12.96 & 0.52 & -0.27 & -0.82 & 0.44 \\
\hline & & Length & $\mathrm{Cm}$ & 143 & 2.12 & 142.8 & 2.28 & 0.2 & 0.14 & 0.89 \\
\hline & & Weight & $\mathbf{K g}$ & 39 & 4.42 & 38 & 2.35 & $\mathbf{1}$ & 0.45 & 0.67 \\
\hline \multirow{8}{*}{ : } & \multirow{3}{*}{$\underset{Z}{Z}$} & $\begin{array}{l}\text { Arms bent from } \\
\text { of pivot / 10sec }\end{array}$ & repetition & 9.2 & 0.45 & 9.4 & 0.55 & -0.2 & -0.63 & 0.54 \\
\hline & & $\begin{array}{l}\text { Arms bent from } \\
\text { hanging / } 10 \mathrm{sec}\end{array}$ & repetition & 8 & 0.71 & 8.6 & 0.55 & -0.6 & -1.5 & 0.17 \\
\hline & & $\begin{array}{l}\text { Push ground with } \\
\text { arms from oblique } \\
\text { laying }\end{array}$ & repetition & 12.4 & 0.55 & 12.4 & 0.55 & $\mathbf{0}$ & $\mathbf{0}$ & 1 \\
\hline & \multirow{3}{*}{ 竭 } & Lift legs from lying & repetition & 10.6 & 0.55 & 10.4 & 0.55 & 0.2 & 0.58 & 0.58 \\
\hline & & Lift legs from hanging & repetition & 7 & $\mathbf{0}$ & 7.2 & 0.45 & -0.2 & -1 & 0.35 \\
\hline & & $\begin{array}{l}\text { Lift back and arms } \\
\text { from lying }\end{array}$ & repetition & 15.2 & 0.45 & 15.4 & 0.55 & -0.2 & -0.63 & 0.54 \\
\hline & \multirow{2}{*}{ 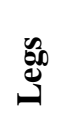 } & Long jump & $\mathrm{Cm}$ & 209 & 1 & 209 & 0.71 & $\mathbf{0}$ & $\mathbf{0}$ & 1 \\
\hline & & Vertical jump & $\mathbf{C m}$ & 40.8 & 1.1 & 41 & 1 & -0.2 & -0.3 & 0.77 \\
\hline \multirow{4}{*}{ 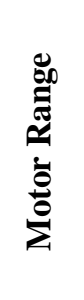 } & & Dome & $\mathrm{Cm}$ & 5.4 & 0.89 & 5.4 & 0.55 & $\mathbf{0}$ & $\mathbf{0}$ & 1 \\
\hline & & nt trunk down forward & $\mathrm{Cm}$ & 12.6 & 0.55 & 12.4 & 0.55 & 0.2 & 0.58 & 0.58 \\
\hline & & Bent arms by degree & Mark & 31.6 & 1.14 & 31 & 1 & 0.6 & 0.88 & 0.4 \\
\hline & & gs backward by degree & Mark & 23 & 2.74 & 21.6 & 2.3 & 1.4 & 0.88 & 0.41 \\
\hline \multirow{3}{*}{ 离 } & & ront straight full twist & Mark & 3.8 & 0.84 & 4 & 0.71 & -0.2 & -0.41 & 0.68 \\
\hline & Str & $\begin{array}{l}\text { aight full twist with turn } \\
\text { around long axis }\end{array}$ & Mark & 3.2 & 0.45 & 3.6 & 0.89 & -0.4 & -0.89 & 0.4 \\
\hline & & Two skills linking & Mark & 2.6 & 0.55 & 3 & 1 & -0.4 & -0.78 & 0.46 \\
\hline
\end{tabular}

T sig. at $0.05=2.36$

Table (2) for statistical significance for basic variables and physical measurements and judges skill level degrees for experimental and control groups before experiment results revel no significant differences between the two groups which means that they are homogeneous

\section{The second pilot study:}

Researcher has identified the most important measurements and tests that measure physical elements (appendix 1) and designed performance evaluation sheet for front acrobatic routine (appendix 2). 
Skill Performance level assessment:

Skill performance level assessment for front acrobatic routine under study was done by judges authorized by Egyptian Gymnastics Federation.

\section{Program duration:}

- Experiment took three months (12 weeks) with 3 sessions per week during the period from June to August 2009.appendix (3)

- The researcher the plyometric exercise training program to develop explosive power for legs. Appendix (3)

Post measurement:

After completion of experiment post measurements carried out of all measurements applied in the same way and conditions that took place in the pre measurement, results were recorded; statistical work done in order to arrive to research results.

Statistical work used:

1 - Mean

2 - standard deviation (SD).

3 - Skewness factor.

4 - Variation coefficient.

$5-\mathrm{T}$ test.
6 - Percentage

7 - Improvement percentage.

8 - Correlation coefficient.

\section{Results and discussion:}

Table (3) for statistical significance for physical measurements and judges skill level degrees for experimental group before and after experiment reveal that :

Existence of statistically significant differences at 0.05 level in all measurements of power, e.g. number of Lift legs from hanging in 10 seconds was 7.00 times before the experiment and reached 9.200 times At the end of the experiment with difference of 2.2 and this difference is significant at at 0.05 level $(\mathrm{t}=11.00)$ and same picture repeated for measurements of explosive power - For example, vertical jump was $40.800 \mathrm{~cm}$ before experiment improved to $50.800 \mathrm{~cm}$ after experiment with difference $10.00 \mathrm{~cm}$ and this difference is significant at $0.05(\mathrm{t}=11.18)$.

For measurements of the motor range improvement is clear in favor of post measurement of this group as $\mathrm{T}$ value were $(9,14.7,12.67,14.0), \quad$ respectively, with statistically significant differences.

Consequent this group was superior in judges skill level evaluation after experiment than before it with significant $\mathrm{T}$ values $(12.55,18.26, \quad 17.42) \quad$ respectively). 
Table (3)

Statistical significance for physical measurements and judges skill level degrees for experimental group before and after experiment

$\mathbf{n}=\mathbf{5}$

\begin{tabular}{|c|c|c|c|c|c|c|c|c|c|c|c|}
\hline \multirow{2}{*}{\multicolumn{3}{|c|}{ Statistical indicators }} & \multirow{2}{*}{ unit } & \multicolumn{2}{|c|}{$\begin{array}{c}\text { Pre- } \\
\text { Measurement }\end{array}$} & \multicolumn{2}{|c|}{$\begin{array}{c}\text { Post- } \\
\text { measurements }\end{array}$} & \multirow{2}{*}{ 莺 } & \multirow{2}{*}{$\mathbf{T}$} & \multirow{2}{*}{ 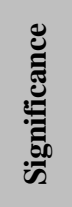 } & \multirow{2}{*}{ 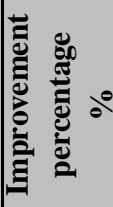 } \\
\hline & & & & Mean & SD & Mean & SD & & & & \\
\hline \multirow{8}{*}{ : } & \multirow{3}{*}{$\underset{z}{Z}$} & $\begin{array}{l}\text { Arms bent from } \\
\text { of pivot / 10sec }\end{array}$ & repetition & 9.20 & 0.45 & 10.80 & 0.45 & 1.60 & $6.53 *$ & 0.00 & 17.39 \\
\hline & & $\begin{array}{l}\text { Arms bent from } \\
\text { hanging / } 10 \mathrm{sec}\end{array}$ & repetition & 8.00 & 0.71 & 10.40 & 0.55 & 2.40 & $6.00 *$ & 0.00 & 30.00 \\
\hline & & $\begin{array}{c}\text { Push ground with } \\
\text { arms from oblique } \\
\text { laying } \\
\end{array}$ & repetition & 12.40 & 0.55 & 14.20 & 0.45 & 1.80 & $9.00 *$ & 0.00 & 14.52 \\
\hline & \multirow{3}{*}{ 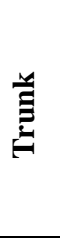 } & Lift legs from lying & repetition & 10.60 & 0.55 & 12.80 & 0.84 & 2.20 & $11.00 *$ & 0.00 & 20.75 \\
\hline & & $\begin{array}{c}\text { Lift legs from } \\
\text { hanging }\end{array}$ & repetition & 7.00 & 0.00 & 9.20 & 0.45 & 2.20 & $11.00 *$ & 0.00 & 31.43 \\
\hline & & $\begin{array}{l}\text { Lift back and arms } \\
\text { from lying }\end{array}$ & repetition & 15.20 & 0.45 & 17.20 & 0.45 & 2.00 & - & - & 13.16 \\
\hline & \multirow{2}{*}{ 包 } & Long jump & $\mathrm{Cm}$ & 209.00 & 1.00 & 222.00 & 2.74 & 13.00 & $8.76^{*}$ & 0.00 & 6.22 \\
\hline & & Vertical jump & $\mathbf{C m}$ & 40.80 & 1.10 & 50.80 & 1.79 & 10.00 & $11.18 *$ & 0.00 & 24.51 \\
\hline \multirow{4}{*}{ 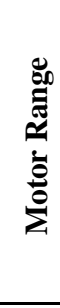 } & & Dome & $\mathbf{C m}$ & 5.40 & 0.89 & 0.90 & 0.22 & -4.50 & $-9.00 *$ & 0.00 & 83.33 \\
\hline & & $\begin{array}{l}\text { Bent trunk down } \\
\text { forward }\end{array}$ & $\mathbf{C m}$ & 12.60 & 0.55 & 16.20 & 0.45 & 3.60 & $14.70 *$ & 0.00 & 28.57 \\
\hline & & ent arms by degree & Mark & 31.60 & 1.14 & 39.20 & 0.45 & 7.60 & $12.67 *$ & 0.00 & 24.05 \\
\hline & & $\begin{array}{l}\text { Legs backward by } \\
\text { degree }\end{array}$ & Mark & 23.00 & 2.74 & 37.00 & 2.74 & 14.00 & $14.00 *$ & 0.00 & 60.87 \\
\hline \multirow{3}{*}{ 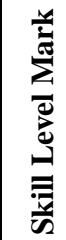 } & & ont straight full twist & Mark & 3.80 & 0.84 & 9.20 & 0.27 & 5.40 & $12.55^{*}$ & 0.00 & 142.11 \\
\hline & & $\begin{array}{l}\text { raight full twist with } \\
\text { turn } \\
\text { around long axis }\end{array}$ & Mark & 3.20 & 0.45 & 8.20 & 0.27 & 5.00 & $18.26 *$ & 0.00 & 156.25 \\
\hline & & Two skills linking & Mark & 2.60 & 0.55 & 9.00 & 0.35 & 6.40 & $17.42 *$ & 0.00 & 246.15 \\
\hline
\end{tabular}

T sig. at 0.05 level $=2.766$

From table (4) for statistical significance for physical measurements and judges skill level degrees for control group before and after experiment

the level of performance skill for exercises subject of research in the control group before and after the experiment, it is clear that there is statistically significant differences at 0.05 level of $33.333 \%$ of total measurements, While the rest $(66.66 \%)$ did not show statistically significant differences at 0.05 level even though this mean differences ware in favor of post measurements, which means that there was improvement but not met significance criteria. 
Table (4)

Statistical significance for physical measurements and judges skill level degrees for control group before and after experiment

$\mathbf{n}=\mathbf{5}$

\begin{tabular}{|c|c|c|c|c|c|c|c|c|c|c|c|c|}
\hline \multirow{2}{*}{\multicolumn{4}{|c|}{ Statistical indicators }} & \multirow{2}{*}{ Unit } & \multicolumn{2}{|c|}{$\begin{array}{c}\text { Pre- } \\
\text { measurement }\end{array}$} & \multicolumn{2}{|c|}{$\begin{array}{c}\text { Post- } \\
\text { measurements }\end{array}$} & \multirow{2}{*}{ 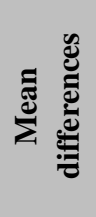 } & \multirow{2}{*}{$\mathbf{T}$} & \multirow{2}{*}{ 总 } & \multirow{2}{*}{ 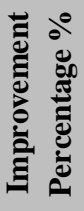 } \\
\hline & & & & & Mean & SD & Mean & SD & & & & \\
\hline \multirow{8}{*}{\multicolumn{2}{|c|}{ 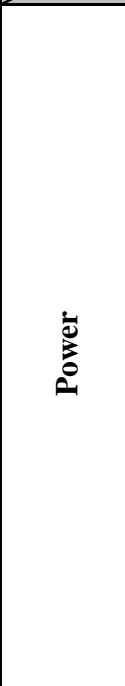 }} & \multirow{3}{*}{$\underset{z}{Z}$} & $\begin{array}{l}\text { Arms bent from } \\
\text { of pivot / 10sec }\end{array}$ & repetition & 9.40 & 0.55 & 9.80 & 0.84 & 0.40 & 1.63 & 0.18 & 4.26 \\
\hline & & & $\begin{array}{l}\text { Arms bent from } \\
\text { hanging / } 10 \mathrm{sec}\end{array}$ & repetition & 8.60 & 0.55 & 7.80 & 0.45 & -0.80 & -2.14 & 0.10 & -9.30 \\
\hline & & & $\begin{array}{c}\text { Push ground with } \\
\text { arms from oblique } \\
\text { laying }\end{array}$ & repetition & 12.40 & 0.55 & 12.20 & 0.45 & -0.20 & -1.00 & 0.37 & -1.61 \\
\hline & & \multirow{3}{*}{ E } & Lift legs from lying & repetition & 14.40 & 0.55 & 11.20 & 0.45 & 0.80 & $4.00 *$ & 0.02 & 7.69 \\
\hline & & & $\begin{array}{l}\text { Lift legs from } \\
\text { hanging }\end{array}$ & repetition & 7.20 & 0.45 & 7.60 & 0.55 & 0.40 & 1.63 & 0.18 & 5.56 \\
\hline & & & $\begin{array}{c}\text { Lift back and arms } \\
\text { from lying } \\
\end{array}$ & repetition & 15.40 & 0.55 & 15.60 & 0.55 & 0.20 & 1.00 & 0.37 & 1.30 \\
\hline & & \multirow{2}{*}{ 包 } & Long jump & $\mathbf{C m}$ & 209.90 & 0.71 & 209.60 & 0.55 & 0.60 & 1.50 & 0.21 & 0.29 \\
\hline & & & Vertical jump & $\mathbf{C m}$ & 41.00 & 1.00 & 42.80 & 1.30 & 1.80 & $4.81 *$ & 0.01 & 4.39 \\
\hline \multirow{4}{*}{$\sum^{\frac{e}{0}}$} & \multirow{4}{*}{ 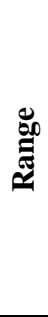 } & & Dome & $\mathbf{C m}$ & 5.40 & 0.55 & 0.80 & 0.45 & -2.45 & -0.55 & 0.07 & -11.11 \\
\hline & & & $\begin{array}{l}\text { Bent trunk down } \\
\text { forward }\end{array}$ & $\mathbf{C m}$ & 12.40 & 0.55 & 13.40 & 0.55 & 1.00 & - & - & 8.06 \\
\hline & & & ent arms by degree & Mark & 31.00 & 1.00 & 31.00 & 0.55 & 0.60 & 2.45 & 0.07 & 1.94 \\
\hline & & & $\begin{array}{l}\text { Legs backward by } \\
\text { degree }\end{array}$ & Mark & 21.60 & 2.30 & 27.80 & 2.59 & 6.20 & 3.50* & 0.02 & 28.70 \\
\hline \multirow{3}{*}{ 党 } & \multirow{3}{*}{ 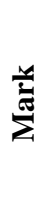 } & & nt straight full twist & Mark & 4.00 & 0.71 & 5.60 & 0.55 & 1.60 & 7.00* & 0.02 & 40.00 \\
\hline & & & $\begin{array}{l}\text { aight full twist with } \\
\text { rn around long axis }\end{array}$ & Mark & 3.60 & 0.89 & 4.60 & 0.89 & 1.00 & - & - & 27.78 \\
\hline & & & Two skills linking & Mark & 3.00 & 1.00 & 4.80 & 0.76 & 1.80 & $5.31 *$ & 0.01 & 60.00 \\
\hline
\end{tabular}

T sig. at 0.05 level $=2.766$

Table (5) and Figures (1 to 3) for physical measurements and judges skill level degrees between control and experimental groups after experiment results reveal the superiority of experimental group in all measurements and test applied :(power - explosive power motor range) which led to improvement in Judges' score for skill level $(\mathrm{T}=13.15,8.61)$ respectively.
Accordingly, researcher can attribute experimental group improvement compared with what control group achieved to the program nature for experimental group , which is based on using plyometric exercise to develop explosive power as described in appendix 
Table (5)

Statistical significance for physical measurements and judges skill level degrees between control and experimental groups after experiment

$\mathbf{n}=\mathbf{5}$

\begin{tabular}{|c|c|c|c|c|c|c|c|c|c|c|c|c|c|}
\hline \multirow{2}{*}{\multicolumn{3}{|c|}{ Statistical indicators }} & \multirow{2}{*}{ Unit } & \multicolumn{2}{|c|}{$\begin{array}{c}\text { Experimental } \\
\text { group } \\
n=5\end{array}$} & \multicolumn{2}{|c|}{$\begin{array}{c}\text { Control } \\
\text { Group } \\
n=5\end{array}$} & \multirow{2}{*}{$\sum^{ \pm}$} & \multirow{2}{*}{$\mathbf{T}$} & \multirow{2}{*}{ Sig. } & \multirow{2}{*}{$\varepsilon^{2}$} & \multirow{2}{*}{ 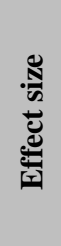 } & \multirow{2}{*}{ 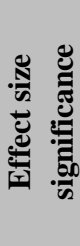 } \\
\hline & & & & Mean & SD & Mean & SD & & & & & & \\
\hline \multirow{8}{*}{ : } & \multirow{3}{*}{$\ddot{z}$} & $\begin{array}{c}\text { Arms bent } \\
\text { from of pivot / } \\
\text { 10sec }\end{array}$ & repetition & $\stackrel{10}{\stackrel{8}{0}}$ & $\stackrel{n}{\circ}$ & $\begin{array}{l}\stackrel{0}{\infty} \\
\text { a }\end{array}$ & $\stackrel{+}{\infty}$ & هُ & $\stackrel{*}{*}$ & $\stackrel{n}{0}$ & $\stackrel{\nabla}{\ominus}$ & $\stackrel{\rho}{\stackrel{\vartheta}{+}}$ & 해 \\
\hline & & $\begin{array}{c}\text { Arms bent } \\
\text { from hanging / } \\
10 \mathrm{sec}\end{array}$ & repetition & $\stackrel{?}{\stackrel{9}{\bullet}}$ & ñn & 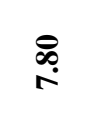 & $\stackrel{n}{9}$ & $\begin{array}{l}\stackrel{8}{0} \\
\text { i }\end{array}$ & 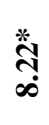 & $\stackrel{8}{\circ}$ & $\stackrel{\infty}{\circ}$ & $\begin{array}{l}\text { กิ } \\
\text { in }\end{array}$ &  \\
\hline & & $\begin{array}{l}\text { Push ground } \\
\text { with arms from } \\
\text { oblique laying }\end{array}$ & repetition & $\begin{array}{l}\text { సิ } \\
\stackrel{\Xi}{ }\end{array}$ & $\stackrel{10}{9}$ & ત્ & $\stackrel{n}{8}$ & $\underset{\text { ) }}{\stackrel{\text { i }}{1}}$ & مُ & $\stackrel{8}{\stackrel{0}{0}}$ & $\stackrel{\circ}{\circ}$ & $\underset{+}{\stackrel{\gamma}{+}}$ & . \\
\hline & \multirow{3}{*}{ E气 } & $\begin{array}{l}\text { Lift legs from } \\
\text { lying }\end{array}$ & repetition & $\begin{array}{l}\underset{\infty}{0} \\
\text { త్ }\end{array}$ & $\stackrel{+}{\oplus}$ & 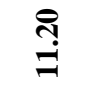 & $\stackrel{10}{0}$ & $\stackrel{8}{\circ}$ & ले & $\vec{\theta}$ & $\stackrel{\nabla}{\Delta}$ & ले & 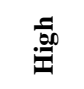 \\
\hline & & $\begin{array}{l}\text { Lift legs from } \\
\text { hanging }\end{array}$ & repetition & ă & $\stackrel{10}{\stackrel{+}{0}}$ & بִ & ஜñ &  & 葛 & $\stackrel{8}{\stackrel{8}{0}}$ & $\stackrel{0}{0}$ & ஸે & : \\
\hline & & $\begin{array}{l}\text { Lift back and } \\
\text { arms from } \\
\text { lying }\end{array}$ & repetition & ત્ని & $\stackrel{10}{\stackrel{0}{0}}$ & 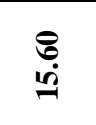 & 哓 & 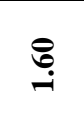 & 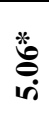 & $\stackrel{8}{0}$ & $\stackrel{0}{\stackrel{0}{0}}$ & ભે & 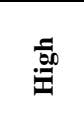 \\
\hline & \multirow{2}{*}{ 氜 } & Long jump & $\mathbf{C m}$ & $\begin{array}{l}\text { ๕. } \\
\text { ส̇ }\end{array}$ & $\underset{i}{i}$ & ๕̊. & 䛇 & 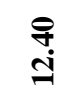 & $\stackrel{\%}{\alpha}$ & $\stackrel{8}{\circ}$ & $\stackrel{\Omega}{\Theta}$ & 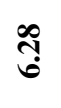 & 㯊 \\
\hline & & Vertical jump & $\mathbf{C m}$ & ஜ̊ & $\stackrel{7}{7}$ & $\begin{array}{l}\stackrel{8}{0} \\
\stackrel{+}{+}\end{array}$ & ঙ̊. & $\underset{\infty}{\varnothing}$ & $\stackrel{*}{*} \stackrel{0}{\stackrel{0}{\circ}}$ & $\stackrel{8}{8}$ & $\stackrel{\infty}{\circ}$ & $\overrightarrow{7}$ & 可 \\
\hline \multirow{4}{*}{ 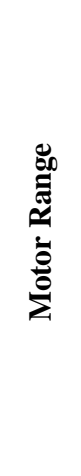 } & & Dome & $\mathbf{C m}$ & $\stackrel{8}{\circ}$ & $\stackrel{\dddot{̣}}{\oplus}$ & $\stackrel{\infty}{\stackrel{\infty}{+}}$ & $\stackrel{n}{\oplus}$ & बे. &  & $\stackrel{8}{\circ}$ & $\hat{a}$ & $\stackrel{\varrho}{\xi}$ & 武 \\
\hline & & $\begin{array}{l}\text { ent trunk down } \\
\text { forward }\end{array}$ & $\mathbf{C m}$ & శึ్ & $\stackrel{10}{+:}$ & $\stackrel{q}{\stackrel{9}{7}}$ & مُ & 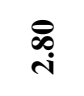 & $\begin{array}{l}* \\
1 \\
\infty \\
\infty \\
\infty\end{array}$ & $\stackrel{8}{\circ}$ & $\bar{a}$ & $\begin{array}{l}\vec{\Xi} \\
\dot{10}\end{array}$ & 武 \\
\hline & Ben & t arms by degree & Mark & लें & $\stackrel{10}{\stackrel{0}{0}}$ & ले & ing & ํㅜㅁ & 节 & $\stackrel{8}{\circ}$ & ڤे & ণิ: & 疍 \\
\hline & & $\begin{array}{l}\text { gs backward by } \\
\text { degree }\end{array}$ & Mark & 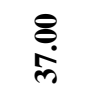 & $\underset{i}{i}$ & సি & 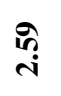 & $\stackrel{\text { กิ }}{\alpha}$ & 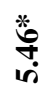 & $\stackrel{8}{8}$ & $\stackrel{\vartheta}{\stackrel{\theta}{\theta}}$ & $\stackrel{\text { fo }}{\text { மे }}$ & 㱐 \\
\hline \multirow{3}{*}{ 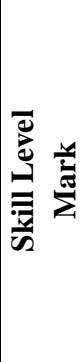 } & & $\begin{array}{l}\text { ont straight full } \\
\text { twist }\end{array}$ & Mark & ๙ૈ? & $\stackrel{\widehat{N}}{\Theta}$ & $\begin{array}{l}8 \\
\text { in } \\
\end{array}$ & $\stackrel{n}{n}$ & $\begin{array}{l}\text { छ̣ } \\
\dot{m}\end{array}$ & $\frac{*}{2}$ & $\stackrel{8}{8}$ & $\stackrel{\circ}{\circ}$ & $\bar{\infty}$ & 해요 \\
\hline & & $\begin{array}{l}\text { raight full twist } \\
\text { th turn around } \\
\text { long axis }\end{array}$ & Mark & స్తి & $\stackrel{\widehat{\jmath}}{\varrho}$ & $\stackrel{8}{\stackrel{8}{+}}$ & $\stackrel{\text { ळे }}{\stackrel{0}{0}}$ & غ. & $\begin{array}{l}\frac{*}{6} \\
\infty \\
\infty\end{array}$ & $\stackrel{8}{\stackrel{8}{\theta}}$ & @̊ & $\underset{i}{\sharp}$ & .50 \\
\hline & & vo skills linking & Mark & $\stackrel{8}{0}$ & $\stackrel{n}{\dddot{g}}$ & 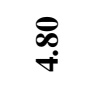 & $\stackrel{0}{0}$ & $\underset{\text { ণิ }}{+}$ & $\stackrel{*}{\stackrel{*}{\Xi}}$ & $\stackrel{8}{\varrho}$ & $\stackrel{+}{0}$ & $\stackrel{0}{i}$ & 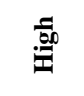 \\
\hline
\end{tabular}

T sig. at 0.05 level $=2.306$ 
Fig (1)

Power measurements mean values for experimental and control groups after experiment
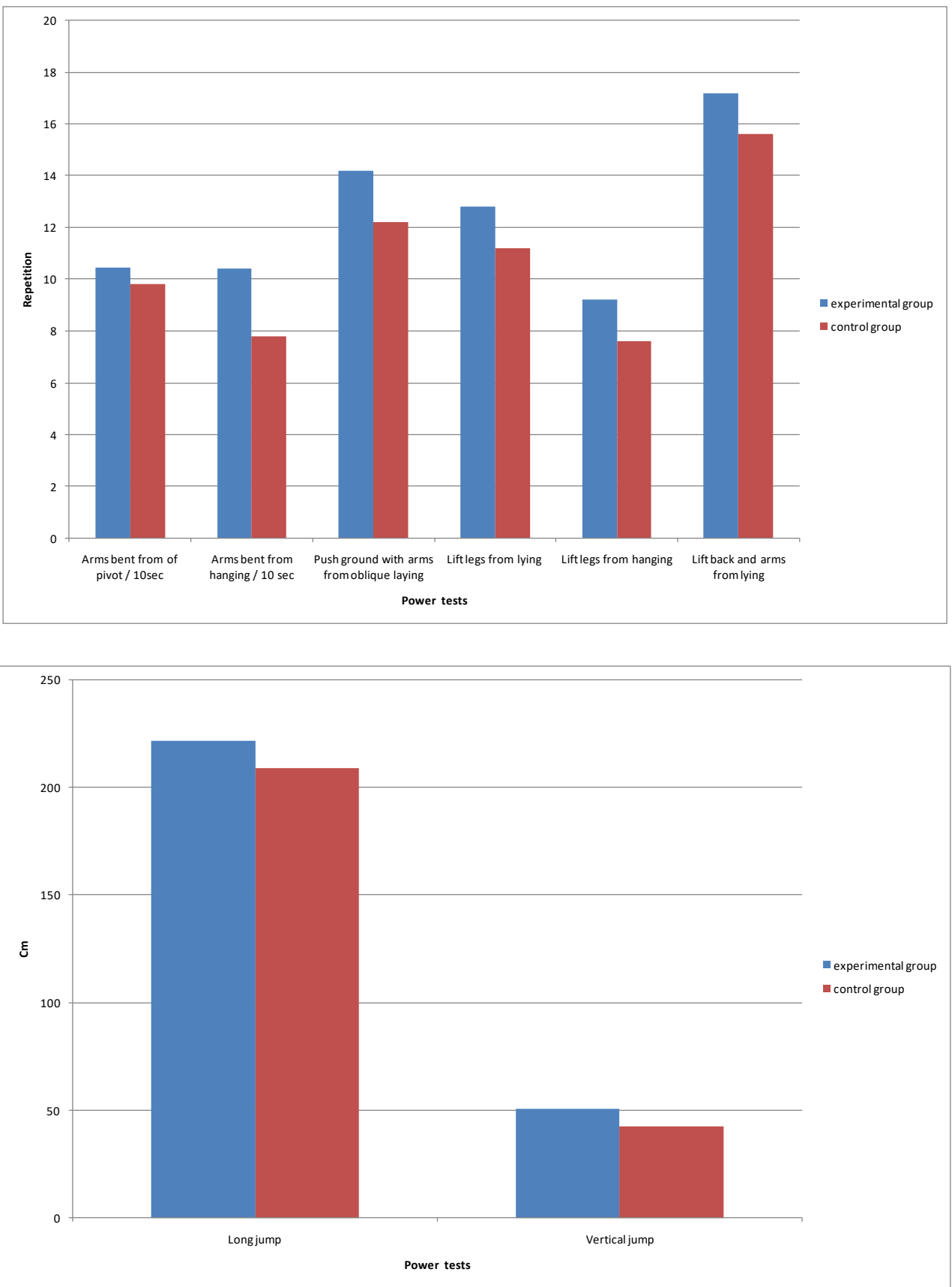
Fig (2)

Motor range measurements mean values for experimental and control groups after experiment
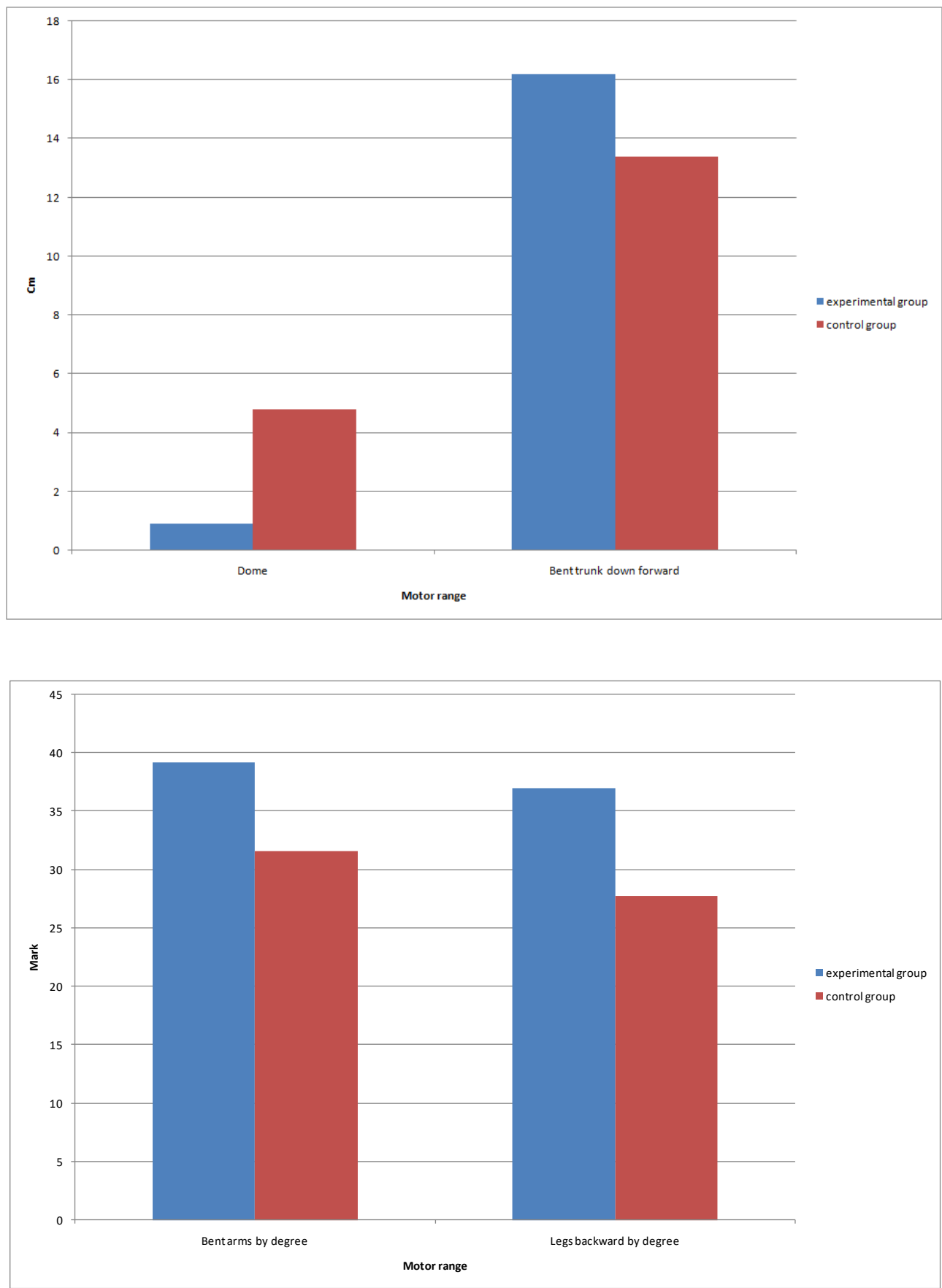
Fig (3)

Skill level score mean values for experimental and control groups after experiment

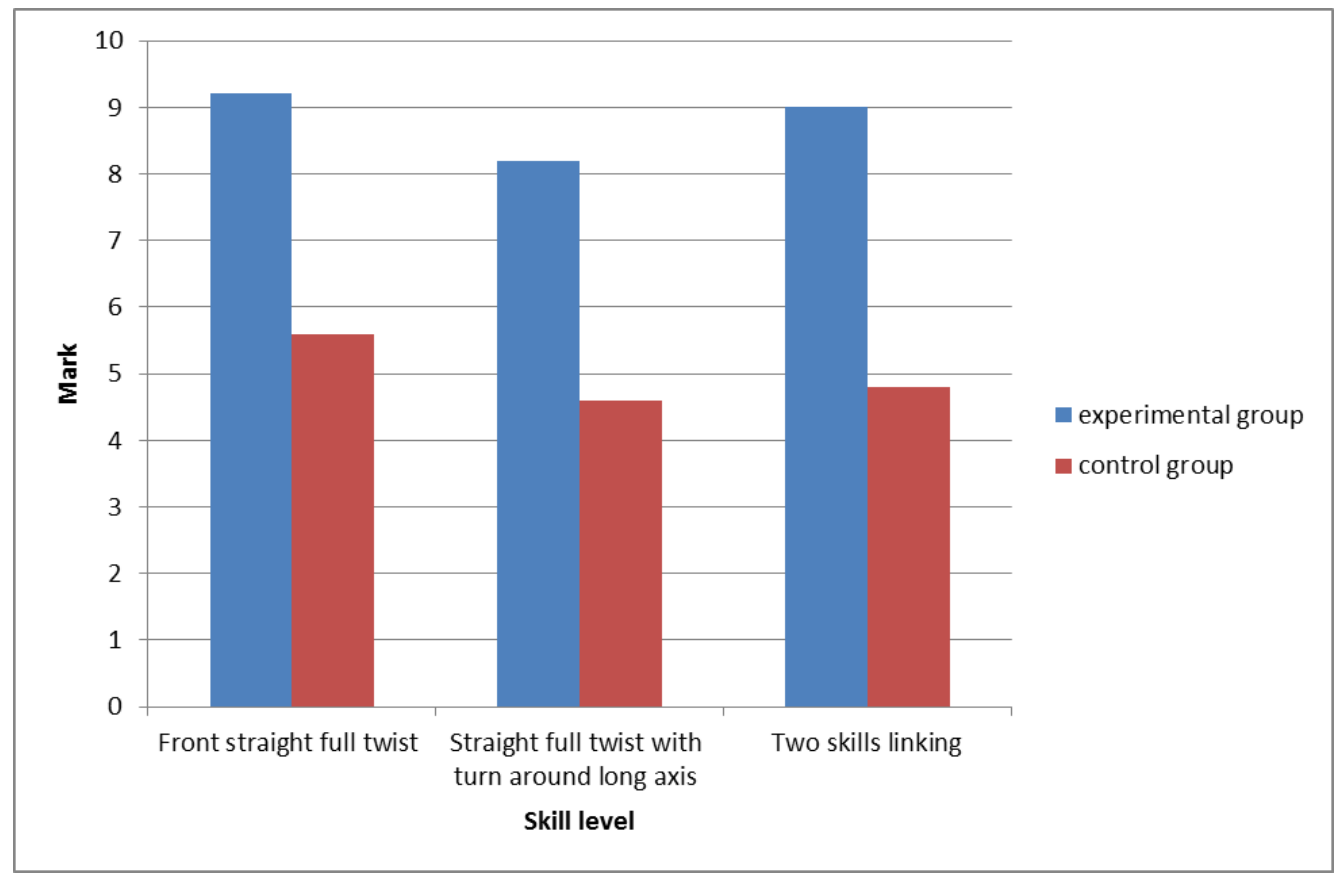

Tale (6) results show that improvement percentage for experimental group was higher than that of control groups in all measurements and tests applied.

Table (6)

improvement percentage for research groups after experiment

\begin{tabular}{|c|c|c|c|c|c|c|c|c|c|c|c|c|c|c|c|}
\hline 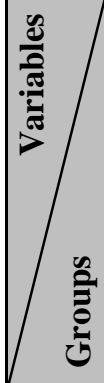 & 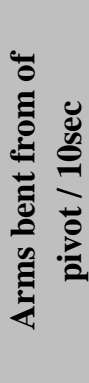 & 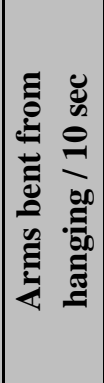 & 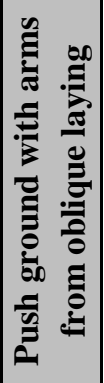 &  & 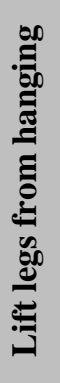 & 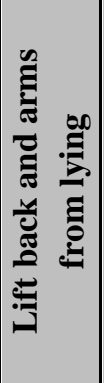 & 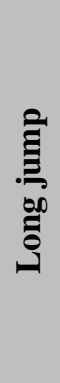 & 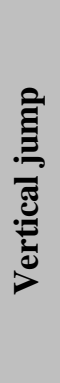 & 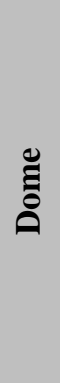 & 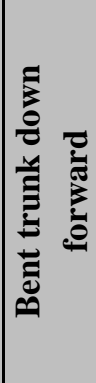 & 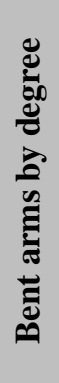 & 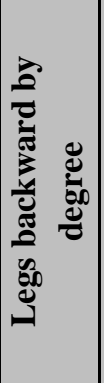 & 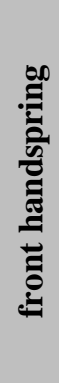 & 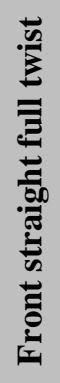 & 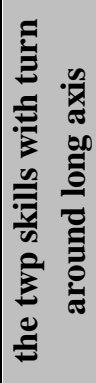 \\
\hline 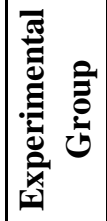 & ले & 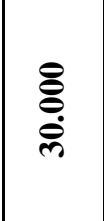 & 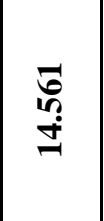 & $\frac{\text { If }}{\stackrel{8}{8}}$ & 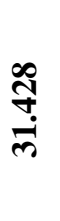 & $\frac{n}{n}$ &  & $\begin{array}{l}\stackrel{g}{8} \\
\dot{d} \\
\text { d }\end{array}$ & $\begin{array}{l}8 \\
8 \\
8\end{array}$ & 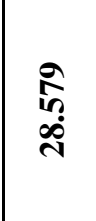 & 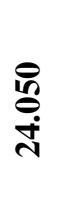 & 홇 & 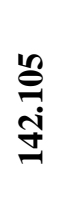 & $\begin{array}{l}m \\
\stackrel{m}{\Xi} \\
\stackrel{n}{n}\end{array}$ & $\begin{array}{l}\text { 今) } \\
\text { fं } \\
\text { Jे }\end{array}$ \\
\hline 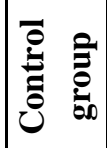 &  & ๙ิ & $\begin{array}{l}\hat{\infty} \\
\text { in } \\
\end{array}$ & ڤ్రి & 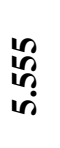 & సొ & 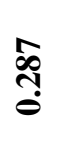 & ঙे & $\exists$ & 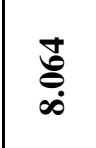 & $\stackrel{n}{\sigma}$ & స̊ & 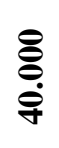 & $\underset{⿱}{\stackrel{N}{N}}$ & $\begin{array}{l}8 \\
8 \\
8 \\
8\end{array}$ \\
\hline
\end{tabular}


Table (7)

Correlation coefficient between skill level and physical measurements and test for overall rserach sample

\begin{tabular}{|c|c|c|c|c|c|}
\hline \multicolumn{2}{|c|}{ Ptatistical indicators } & Unit & $\begin{array}{c}\text { Front } \\
\text { straight full } \\
\text { twist }\end{array}$ & $\begin{array}{l}\text { Straight full } \\
\text { twist with turn } \\
\text { around long } \\
\text { axis }\end{array}$ & $\begin{array}{c}\text { Straight full twist } \\
\text { with turn around } \\
\text { long axis }\end{array}$ \\
\hline \multirow{6}{*}{ Power } & $\begin{array}{l}\text { Arms bent from of } \\
\text { pivot / 10sec }\end{array}$ & repetition & 0.801 & 0.853 & 0.822 \\
\hline & $\begin{array}{l}\text { Arms bent from } \\
\text { hanging / } 10 \text { sec }\end{array}$ & repetition & 0.119 & 0.08 & 0.124 \\
\hline & $\begin{array}{l}\text { Push ground with arms } \\
\text { from oblique laying }\end{array}$ & repetition & 0.8 & 0.88 & 0.822 \\
\hline & Lift legs from lying & repetition & 0.867 & 0.881 & 0.874 \\
\hline & Lift legs from hanging & repetition & 0.908 & 0.932 & 0.939 \\
\hline & $\begin{array}{l}\text { Lift back and arms } \\
\text { from lying }\end{array}$ & repetition & 0.883 & 0.882 & 0.895 \\
\hline \multirow{2}{*}{$\begin{array}{l}\text { Explosive } \\
\text { power }\end{array}$} & Long jump & $\mathrm{Cm}$ & 0.887 & 0.902 & 0.887 \\
\hline & Vertical jump & $\mathrm{Cm}$ & 0.934 & 0.961 & 0.969 \\
\hline \multirow{4}{*}{$\begin{array}{l}\text { Motor } \\
\text { range }\end{array}$} & Dome & $\mathrm{Cm}$ & 0.925 & 0.913 & 0.923 \\
\hline & $\begin{array}{l}\text { Bent trunk down } \\
\text { forward }\end{array}$ & $\mathbf{C m}$ & 0.971 & 0.94 & 0.956 \\
\hline & Bent arms by degree & Mark & 0.921 & 0.935 & 0.941 \\
\hline & $\begin{array}{c}\text { Legs backward by } \\
\text { degree }\end{array}$ & Mark & 0.832 & 0.795 & 0.832 \\
\hline \multirow{3}{*}{$\begin{array}{l}\text { Motor } \\
\text { range }\end{array}$} & Front straight full twist & Mark & - & 0.929 & 0.968 \\
\hline & $\begin{array}{l}\text { Straight full twist with } \\
\text { turn around long axis }\end{array}$ & Mark & 0.929 & - & 0.977 \\
\hline & Two skills linking & Mark & 0.968 & 0.977 & - \\
\hline
\end{tabular}

Table (7) results reveal high correlation between skill level and all physical measurements and tests applied except for bent knee from hanging

\section{Discussion:}

Tables (6), (7), (8) results reveal existence of statistically significant difference between pre and post measurements for physical measurements (power-explosive power motor range) in favor of post measurement and between measurements and improvement percentages for research groups in favor of experimental group. This indicates the effectiveness of plyometric training exercises in the program, where they work in the same direction as muscular work required for of explosive power development required for 
developing acrobatic routine under study, which have positive effect on improving power, motor range, and hence the acrobatic routine, as demonstrated by results of $\mathrm{T}$ test. These results is consistent with Asia Solimon (2005), Hosni Sayyid Ahmad, Hazem Hassan (2003), Mohamed Shawky, and Amr Allah Busatti (2002) Maha Amin (2000), Yasser Ashour (1999), studies results which refers to the preference of plyometric training in improving explosive power, which helped to improve skill performance level under discussion.

Mohammed Jalal (2004: p5) and Osama Abdel Fattah (2004: p9) and Zaky Darwish (1998: p5) Mike Hebert (1991: 22), Moran and Glynn (1990: p96) emphasized on the importance of plyometric training as the best way to develop explosive power where this method has contributed to overcome problems of explosive power development.

Dintiman et al (1998: p122) and Abo Elela Abdelfattah (1997: p22) argues that plyometric training is one of the influential and ideal training methods that could be used in explosive power development and legs muscles' speed.

These results also agreed with Mohammed Jalal (2004) and Ashraf Abdel Elaal and Muna Elsayed. (2003) and Costello (1984) studies results which concluded the effectiveness of plyometric training in improving power.

From what mentioned above, it is possible to conclude that plyometric training program positively affect some physical elements associated with acrobatic routine under discussion. This fulfill first hypothesis.

Second hypothesis : "Plyometric training program for positively affect front acrobatic routine performance level" validation is illustrated by tables (6), (7), (8) which indicate existence of statistically significant difference between pre and post measurements for skill performance level in favor of post measurement and between measurements and improvement percentages for research groups in favor of experimental group. These results show that skill performance level has been improved as a result of development of explosive power and other physical variables through plyometric training program applied.

These results agreed with malaka Elrefaay and the Iman Soliman (1993) Maha Amin (2000), Ashraf Abdelaal and Mona Elsayed. (2003), Khalid Mahmood (2005) Saeed Ghonemy (2008) Ostilo (1983), studies results which all agreed that plyometric training positively affect skill level generally and acrobatic routine under study particularly

This is also consistent with what Mike Hebert (1991: p22) argued that plyometric training considered one of the most important types of exercises that contribute to improvement of some physical abilities, most important of them is explosive power that have an impact on skill performance level of many of jumping and pushing skills.)

Talha Hossam El Din (1997:pp80-9), quoting from Chu that plyometric training is the link between muscle strength and power and that is the main entrance to improve performance level through these tow muscle characteristics, and that plyometric training works to direct this strength in appropriate path to improve performance speed.

\section{Conclusions:}

Based on the results that have been reached and that associated with research objectives and in light of the methodology used and within limits of sample used, researcher reached the following conclusions: 
1- Using plyometric training has effective effect in developing physical abilities and explosive power specially of arms and legs.

2-Development of legs explosive power led to improvement in skill performance level.

3-Development of legs explosive power led to the development of other elements (power), motor range and flexibility for muscle involved in performance skills.

4- The presence of a high correlation between skill performance level and explosive power.

\section{Recommendations:}

In light of the results that have been reached through research; researcher recommends the following:

1-There is necessity to pay attention to plyometric training in gymnastics selection of exercises cope with working muscle contractions methods in different skills performance.

2-Gymnastics trainers should pay interest to change training programs types and use plyometric training method, especially with juniors as it have positive impact on Gymnastics skill performance.

3-Conducting similar studies for different age stages and level for male and female players.

4-Plyometric training program to be included as part of physical education curricula.

5- Preparation of scalable training program suitable for application of different ages to contribute in improving skill performance level of gymnastics ground movements.

6-Attention should be given to conduct training courses for newly graduated coaches working in gymnastics training about "how to develop plyometric training programs on various artistic gymnastics equipment.

\section{References:}

1- Abo Elela Ahmed Abdelfattah (1997): sports training and physiological bases, 1st ed., Arab Thought House, Cairo. (in Arabic Language)

2- Ashraf Abdelaal Maraghy, Mona Elsayed Abdelaal(2003): The effect of using some muscle work methods for development of power on development dynamics of some physical characteristics and of skill performance level of front somersault on pommel horse, published research, Journal of theories and applications, issue (46 ), Faculty of Physical Education for Men, Alexandria University. (in Arabic Language)

3- Asia Ali Solimon (2005): Effect of plyometric training program for development of explosive power on skill performance level for ring jump rhythmic gymnastics, unpublished master thesis, Faculty of Physical Education for Girls, Alexandria University. (in Arabic Language)

4- Costello, o.F (1984) : Using weigh troininf and Plyimetric in to increasing explosive Power for Football. Strength condi.

5- Dinitman. G.. et al (1998) : Sport speed, Second Edition, human kinetics publisher, champagne, Illinois.

6- Hebert, mike (1991) : Insights and strolegies fir minning Volleyball : university of Illinois Pres, Chompaign. Illinois.

7- Heider scheit, B.C et al : Effect is Kinetic Troining on the Shaulder inlernal Rolators The Journal of or Thopedic Sports Physical the ropy. U.S.A 27 ed 1996.

8- Hosni Sayed Ahmed, Hazem Hassan Abdullah (2003): Effect of plyometric training on developing explosive power and improving skill performance of front somersault front followed by front one and a half turn meningococcal on a pommel horse, 
journal of sports Arts and Sciences, Assiut University, issue (17)., vol (2), November. (in Arabic Language)

9- International Gymnastics Federation (2006): International law for gymnastics men's Championships in world championships and Olympic Games and regional and international continental competitions, translated by Salah Askar 2006. (in Arabic Language)

10-Khaled Mohammed, Mandour Saidy (2005): The effect of plyometric training programs on improving landing distance on gymnastics pommel horse for junior gymnasts, unpublished Ph.D. thesis, Faculty of Physical Education, Helwan University. . (in Arabic Language)

11-Maha Mohamed Ahmed Amin (2000): The effect of plyometric training on some biomechanics variables and improving skill performance of front hand somersault, unpublished Ph.D. thesis, Faculty of Physical Education for Girls, Alexandria University. (in Arabic Language)

12-Malaka Ahmad Refai Albulasy, and Iman Soliman Abu Dahab (1993): Impact of running and jump exercises on improving jump approach and takeoff stage and performance level of front hands somersault on pommel horse, scientific production, published research, third research, Alexandria . (in Arabic Language)

13-Medhat Mahmoud El Shafei (2005): The effect of using plyometric training on development of muscle ability and some offensive and defensive skills, in handball, scientific production, research publication, Journal of theories and applications, issue (55), Faculty of Physical Education for Men, Alexandria University. (in Arabic language)

14-Mohamed Mohamed Jalal (2004): Effect of plyometric training on development of power and its relationship with effectiveness of most common some offensive skills fir karate players stage (11- 13) unpublished master thesis, , Faculty of Physical Education for Men, Alexandria. (in Arabic Language)

15-Mohamed Shawky El Sebaai, and Amr Allah Ahmad Busatti (2002): the study of impact drills Albleomtria in Alatjath horizontal and vertical ability level explosive compounds speed of some athletes, the production of scientific research publication, Journal of theories and applications, the number (44) College of Physical Education,
Benin, University of Alexandria. (in Arabic Language)

16-Moron, G \&. Meglynn, G., (1990) : Dynomic of Strength Training Sports and Fitness, Bronn Publishers Dubuque Lowe.

17-Osama Mahmoud Abdel Fattah (2004): The effect of plyometric exercises program to develop muscle power and effectiveness of skills performance of field hockey players, unpublished master thesis, , Faculty of Physical Education, Tanta University. (in Arabic Language)

18-Saeed Mohammed Ghonemy Mohammed (2008): Effect of plyometric training using the on developing explosive to improve skill performance of front somersault on pommel horse, unpublished master thesis, Faculty of Physical Education for Men, Alexandria University. . (in Arabic Language)

19-Talha Hussain Husam al-Din, and Wafaa Salah al-Din, Mostafa Kamel Hamad, Said Abdul Rashid (1997): scientific encyclopedia in training (strength - power - power endurance- flexibility), book publication center, Cairo. (in Arabic Language)

20-Westcott. W. (1995) : Strength Fitness, Physiological Primeples and training technique, 4th ed. Wm. E grown consume, Cautions, imc, Madison.

21-Yasser ElSayed Ashour. (1999): The effect of proposed training program to develop arms muscle power on skill performance level of front somersaults on gymnastics ground movements, Unpublished Master Thesis, Faculty of Physical Education for Men, Cairo, Helwan University. (in Arabic Language)

22-Zaky Mohammed Darwish (1998): Plyometric training (development - concept use), Arab Thought House, Cairo. (in Arabic Language 
